\title{
Aptamer redesigned tRNA is nonfunctional and degraded in cells
}

\author{
DENNIS LEE and WILLIAM H. MCCLAIN ${ }^{1}$ \\ Department of Bacteriology, University of Wisconsin, Madison, Wisconsin 53706-1569, USA
}

\begin{abstract}
An RNA aptamer derived from tRNA ${ }^{\text {Gln }}$ isolated in vitro and a rationally redesigned tRNA ${ }^{\text {Gln }}$ were used to address the relationship between structure and function of tRNA ${ }^{\text {Gln }}$ aminoacylation in Escherichia coli. Two mutant tRNA ${ }^{\text {Gln }}$ sequences were studied: an aptamer that binds 26-fold tighter to glutaminyl-tRNA synthetase than wild-type tRNA ${ }^{\text {Gln }}$ in vitro, redesigned in the variable loop, and a mutant with near-normal aminoacylation kinetics for glutamine, redesigned to contain a long variable arm. Both mutants were tested in a tRNA ${ }^{\text {Gln }}$ knockout strain of $E$. coli, but neither supported knockout cell growth. It was later found that both mutant tRNAs were present in very low amounts in the cell. These results reveal the difference between in vitro and in vivo studies, demonstrating the complexities of in vivo systems that have not been replicated in vitro.
\end{abstract}

Keywords: protein-RNA recognition; in vitro aminoacylation; RNA structure

\section{INTRODUCTION}

The in vitro study of tRNA aminoacylation has revealed many intricacies of the complex process of tRNA recognition by aminoacyl tRNA synthetase (aaRS). However, in vitro studies still fail to account for many observations made from in vivo studies. For example, the aminoacylation levels of certain tRNA mutants are $10^{4}$-fold higher in vivo than in vitro (McClain et al. 1999). This observation alludes to the complexity of in vivo tRNA aminoacylation. In vitro studies do not account for the complex interactions between all of the cellular machinery; generally, they are designed to simplify a complex problem. In vitro techniques are therefore valuable in initiating the study of a complex system like tRNA aminoacylation. In fact, the isolation of tRNA mutants through in vitro selections of RNA aptamers would provide a basis for many mutant studies of tRNA aminoacylation.

The in vitro study of RNA aptamers is a fruitful field of scientific research. Aptamers are small nucleic acid ligands that are isolated through in vitro combinatory library selection methods (Gold et al. 1995). Aptamers have been used in a variety of applications from use as molecular sensors to use in protein inhibitors for drug discovery, and use in membrane permeability studies (Khvorova et al. 1999; Famulok et al. 2000; Cerchia et al. 2002). RNA apta-

Reprint requests to: William H. McClain, Department of Bacteriology, University of Wisconsin, 420 Henry Mall, Madison, WI 53706-1569, USA; e-mail: wmcclain@wisc.edu; fax: (608) 263-0772.

Article and publication are at http://www.rnajournal.org/cgi/doi/ 10.1261/rna.5165804. mers have also presented an opportunity to study RNAprotein interactions, an important aspect in the study of the structure-function relationships in tRNA aminoacylation. The careful in vitro study of RNA aptamers coupled with in vivo analyses would reveal many workings of the tRNA aminoacylation system in the context of the living cell.

A tight binding tRNA $^{\text {Gln }}$ aptamer derived from wild-type tRNA $^{\text {Gln }}$ was isolated through an in vitro library selection method (Stubenrauch 1996), and it was found that the majority of the tight binding phenotype was due to the AGGU sequence in the variable loop (Bullock et al. 2000). A mutant tRNA ${ }^{\text {Gln }}$ containing four mutant bases in the variable loop (GlnAGGU tRNA ${ }^{\text {Gln }}$ mutant; Fig. 1B) was therefore synthesized through in vitro transcription and found to have a $K_{d}$ for binding to glutaminyl-tRNA synthetase (GlnRS) that was 26-fold lower than that of wild-type tRNA $^{\text {Gln }}$ in vitro (Bullock et al. 2000). The substantially tighter binding was attributed to a rearrangement in the complex core of tRNA ${ }^{\mathrm{Gln}}$. The crystal structure of the GlnRS-GlnAGGU complex (Bullock et al. 2000) showed that the bases (AGGU) in the tRNA ${ }^{\text {Gln }}$ mutant are stacked more efficiently than in wild-type $\mathrm{tRNA}^{\mathrm{Gln}}$ in which the variable loop bases U47 and U46 are flipped out of the complex core base stacking. The GlnAGGU mutant tRNAGln suggests that stabilization of the tRNA tertiary structure as opposed to optimization of RNA-protein contacts can strengthen GlnRS-tRNA ${ }^{\text {Gln }}$ binding (Bullock et al. 2000). In another mutant, a tRNA ${ }^{\text {Gln }}$ with a serine tRNA long variable loop (Fig. 1C), and a cytosine at position 44 (GlnQSerC44) was synthesized. GlnQSerC44 was shown to be folded properly and aminoacylated in vitro, although the 
A
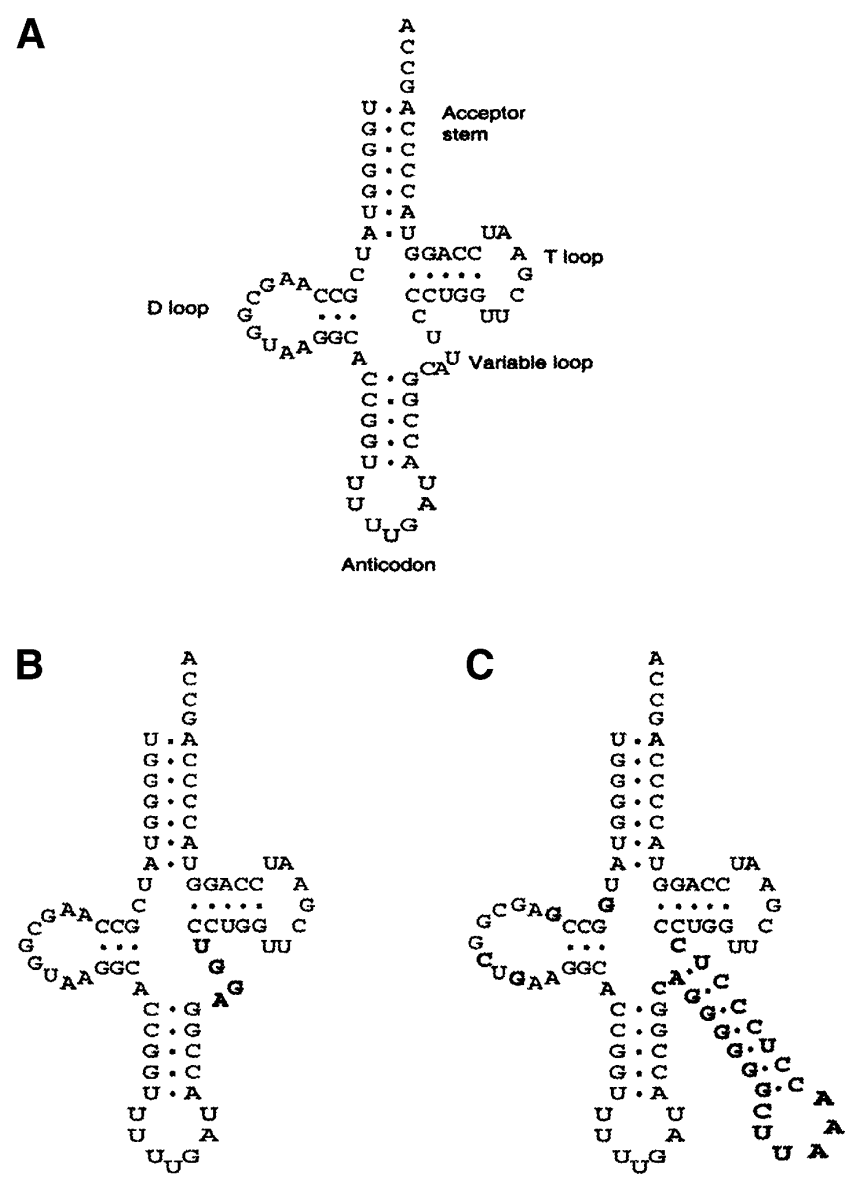

FIGURE 1. Cloverleaf structures of $(A)$ wild-type tRNA ${ }^{\mathrm{Gln}} ;(B)$ the GInAGGU tRNA mutant (Bullock et al. 2000); and $(C)$ the GInQSerC44 tRNA mutant (Nissan et al. 1999). Nucleotides that differ from the wild-type tRNA ${ }^{\text {Gln }}$ sequence are shown as bold letters on the tRNA mutant structures.

$k_{\text {cat }} / K_{m}$ was reduced slightly (fourfold) relative to wild-type tRNA $^{\text {Gln }}$ (Nissan et al. 1999). The aminoacylation kinetics show the capacity of GlnRS to tolerate large differences in the tertiary core of the tRNA while still retaining near wildtype substrate properties in vitro.

Below, we investigate the behavior of the mutants GlnAGGU and GlnQserC44 in the Glns114 tRNA Gln $^{\text {ng }}$ knockout strain of E. coli (W.H. McClain, unpubl.). The Gln 1114 knockout strain lacks all chromosomal copies of the tRNA ${ }^{\mathrm{Gln}}$ genes. Because tRNA ${ }^{\mathrm{Gln}}$ is required for cell survival, the knockout strain is supported by a maintenance plasmid containing a wild-type tRNA ${ }^{\text {Gln }}$ gene, whose expression is under the control of the araCPBAD promoter, which is induced by the presence of arabinose and turned off by the presence of glucose. The properties of these maintenance plasmids have been described (Gabriel and McClain 2001). The two mutant tRNA genes were constructed and constitutively expressed from a high copy test plasmid pGFIB (Masson and Miller 1986) in the Gln 1114 knockout strain. The phenotypes of the two mutants were tested by growing the knockout cells on glucose-containing media, so growth of the cells would be solely dependent on the activity of the tRNA genes expressed from the constitutive test plasmid, given that expression of the wild-type tRNA ${ }^{\text {Gln }}$ gene is turned off in the presence of glucose.

\section{RESULTS AND DISCUSSION}

The function of two tRNA mutants was studied: GlnAGGU (Bullock et al. 2000), a mutant tRNA ${ }^{\text {Gln }}$ containing four (AGGU) instead of the wild-type five (CAUUC) nucleotides in its variable loop (Fig. 1B); and GlnQSerC44 (Nissan et al. 1999), a tRNA ${ }^{\text {Gln }}$ rationally redesigned to contain a tRNA ${ }^{\text {Ser }}$ variable loop, a cytosine at position 44 , and changes at positions $9,13,20$, and $20 \mathrm{~b}$ in the $\mathrm{D}$ stem and loop to accommodate the large variable region (Fig. 1C). The synthetically assembled tRNA genes were ligated into a highcopy pGFIB plasmid. The plasmids expressing the mutant tRNA ${ }^{\text {Gln }}$ genes were electroporated separately into LB liquid cultures of $G \ln \Delta 114$ tRNA ${ }^{\text {Gln }}$ knockout cells where expression of wild-type tRNA ${ }^{\mathrm{Gln}}$ from the regulatable maintenance plasmid is repressed; neither mutant supported growth after $72 \mathrm{~h}$ of incubation at $37^{\circ} \mathrm{C}$ (data not shown). While the $G \ln \Delta 114$ knockout cells did contain the maintenance plasmid, growth as a result of leakiness of the araCpBAD promoter is not a concern because no growth was detected.

The growth phenotype of $G \ln \Delta 114$ knockout cells containing the mutant tRNA genes GlnAGGU and GlnQSerC44 was also determined in a glucose strip plate assay (Gabriel and McClain 2001; Moulinier et al. 2001). Cells transformed with test plasmid pSU81 constitutively expressing each mutant tRNA gene separately were streaked across a plate containing arabinose, which induces expression of the wild-type tRNA ${ }^{\text {Gln }}$ gene from the maintenance plasmid. A strip of sterile paper was then saturated with glucose, which inhibits expression of wild-type tRNA ${ }^{\mathrm{Gln}}$ from the maintenance plasmid, and placed on the surface of the plate. The positive control streak of wild-type tRNA ${ }^{\text {Gln }}$ constitutively expressed from pSU81 showed growth through the entire streak, while the GlnAGGU, GlnQSerC44, and a negative control only grew at the edges of the plates, as the tRNA genes that were constitutively expressed were inactive and expression of the wild-type tRNA ${ }^{\text {Gln }}$ from the maintenance plasmid was inhibited by the glucose strip (Fig. 2). Again, growth as a result of leaky expression of wild-type tRNA ${ }^{\text {Gln }}$ from the maintenance plasmid is not a concern because it would result in growth of the test streaks and negative control near the glucose strip. This was not the case.

To further investigate the no-growth phenotype of the two mutant tRNAs, a Northern analysis was performed ascertaining the amount of the mutant tRNAs GlnAGGU and GlnQSerC44 in transformed E. coli XAC/A16 wild-type cells containing all chromosomal tRNA ${ }^{\text {Gln }}$ gene copies. Extracted RNAs were hybridized to radioactive probes complementary to the anticodon and part of the respective variable 


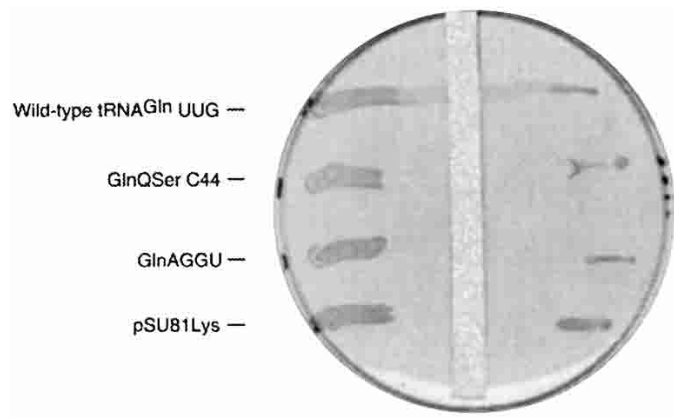

FIGURE 2. Glucose strip test of GInAGGU and GInQSerC44 growth properties in Glns 114 tRNA ${ }^{\text {Gln }}$ knockout cells. Glns 114 cells are supported by pSAD plasmid whose expression of the wild-type tRNA ${ }^{\text {Gln }}$ gene is repressed by glucose, and pSU81 plasmid constitutively expressing wild-type tRNA ${ }^{\text {Gln }}$, GInAGGU, GInQSerC44, and amber tRNA ${ }^{\text {Lys }}$.

loop regions of wild-type $\mathrm{tRNA}^{\mathrm{Gln}}$, GlnQSerC44, and GlnAGGU in a Northern blot. A preliminary film showed neither mutant tRNA ${ }^{\mathrm{Gln}}$ was present. Upon prolonged exposure, the probe for the GlnAGGU tRNA mutant revealed a tRNA-size band in both the GlnAGGU and the pGFIB negative control lane (Fig. 3), suggesting the probe was crosshybridizing to wild-type tRNA ${ }^{\text {Gln }}$ derived from the chromosome. Even so, the presence of the GlnAGGU mutant tRNA would have been detectable as an increase in band intensity because GlnAGGU was expressed from a high-copy plasmid. The comparable intensities of the negative control band and the GlnAGGU band indicate the mature form GlnAGGU mutant tRNA is either not present or is present in small amounts.

Northern signals from GlnQSerC44 also appeared after prolonged exposure, showing two bands that ran slower than wild-type tRNA ${ }^{\mathrm{Gln}}$ (Fig. 3), one in the position of tRNA $^{\text {Gln }}$ with a longer variable loop, and the other typical of precursor tRNA. The slower migrating band in the GlnQSerC44 lane may reflect a defect in processing of the GlnQSerC44 precursor tRNA. The faster migrating band does indicate that there is some mature GlnQSerC44 tRNA in the cell. However, the considerably reduced intensity of the GlnQSerC44 tRNA band relative to the intensity of plasmid expressed wild-type tRNA ${ }^{\text {Gln }}$ suggests that there is not enough mature GlnQSerC44 tRNA to support $G \ln \Delta 114$ cell growth.

The apparent absence of the GlnAGGU mutant and the low intensity bands shown in the GlnQSerC44 lane suggests that both tRNA ${ }^{\mathrm{Gln}}$ mutants and their precursor tRNAs are degraded by cellular RNases. The tertiary core of GlnAGGU is stacked more efficiently than wild-type tRNA ${ }^{\mathrm{Gln}}$ when bound to GlnRS. However, it is unclear that free, unbound GlnAGGU tRNA, which is recognized in the cell by cellular RNA processing, tRNA synthesis, and degradation machinery, has the same efficient stacking in its tertiary core relative to that in the GlnRS-tRNA ${ }^{\text {Gln }}$ complex. Furthermore, the accumulation of precursor molecules of the mutant
tRNA GlnQSerC44 points to the difficulties the cell has in processing this peculiar molecule (Altman 1971). Although thermal melting analyses have shown that the GlnQSerC44 mutant tRNA is well folded as a class II-like fold in vitro (Nissan et al. 1999), the structure may still be unusual with respect to the in vivo tRNA synthesis and/or stability processes. Processing of tRNAs by RNase $\mathrm{P}$ is exquisitely sensitive to the structure of substrate precursor tRNA ${ }^{\text {Gin }}$; the tRNA ${ }^{\text {Gln }}$ portion of the precursor is folded into its canonical tertiary L-shaped form (Seidman et al. 1974; McClain and Seidman 1975). The sensitivity of RNase P to GlnQSerC44 is indicative of the high level of specificity in in vivo processing and degradation of tRNA ${ }^{\text {Gln }}$. Indeed, in vivo studies of 10 separate mutants have shown that single replacements of Watson-Crick G-C base pairs with A-C or G-U mispairs throughout $\mathrm{tRNA}^{\mathrm{Gln}}$ can cause the cell to degrade the tRNA, while subsequent changes of A-C or G-U mispairs to Watson-Crick base pairs prevents tRNA degradation (McClain and Seidman 1987).

All samples showed tRNA size bands when probed by the wild-type $\mathrm{tRNA}^{\mathrm{Gln}}$ probe, as expected, because wild-type tRNA $^{\text {Gln }}$ is expressed from the chromosomal tRNA ${ }^{\text {Gln }}$ genes of the wild-type XAC/A16 strain of E. coli used in the Northern analysis.

In an attempt to isolate a stable and functional tRNA $\mathrm{Gln}^{\mathrm{ln}}$ mutant with four nucleotides in the variable loop, a library was constructed by randomizing the four variable loop nucleotides of the GlnAGGU mutant tRNA. The randomized tRNAs were assembled using a one-step synthesis method (Choi et al. 2002), ligated into the pGFIB plasmid, and introduced into Gln $\Delta 114$ tRNA $^{\text {Gln }}$ knockout cells by electroporation. The experiment did not yield any growthsupporting mutants among $5 \times 10^{10}$ independent clones tested. Thus, the library, which contained 256 possible sequences, was saturated and did not contain an active tRNA.

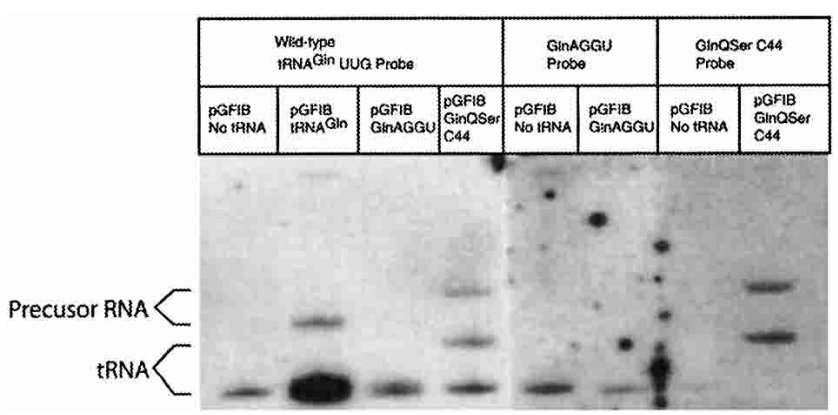

FIGURE 3. Northern analysis of GInAGGU and GInQSerC44 mutant tRNAs. Low molecular weight RNAs were prepared from $X A C / A 16$ cells expressing the plasmids pGFIB, pGFIB tRNA ${ }^{\mathrm{Gln}}$, pGFIB GInAGGU, or pGFIB GInQSerC44. The high background on the films on the right reflects prolonged exposure. Identification of tRNA and precursor tRNA bands were based on gel mobility (Comer et al. 1974; Seidman et al. 1974; McClain and Seidman 1987). The probes for GInQSerC44 showed precursor tRNAs, while the probes for GInAGGU crosshybridized with wild-type tRNA $^{\text {Gln }}$ transcribed from the $X A C / A 16$ chromosome, and showed no signal of overexpressed GInAGGU tRNA. 


\section{CONCLUDING REMARKS}

The absence of both GlnAGGU and GlnQSerC44 in growing cells reveals the distinctions between in vitro and in vivo studies. Although T7 phage tRNA transcripts of both GlnAGGU and GlnQSerC44 were aminoacylated in vitro with purified GlnRS, neither tRNA was present in sufficient amounts in vivo to support knockout cell growth. In vitro studies can give valuable insights into the aminoacylation reaction that are difficult to obtain in vivo. Complicated systems can be broken down to individual parts and studied separately for deeper understanding. Nonetheless, the ultimate goal is to understand how biological systems work in vivo. It is therefore imperative that results from in vitro studies are tested in vivo before conclusions about a system are solidified. The complexities of in vivo systems sometimes makes them difficult to study; however, in vitro systems may excessively simplify a system, leaving out key points. It is therefore essential that both in vivo and in vitro studies are employed to understand the aminoacylation system.

\section{MATERIALS AND METHODS}

\section{Bacterial strains and plasmids}

The following strains and plasmids have been described. E. coli $X A C / A 16$ ( lacpro, nalA, rif, $\arg E_{a m} / F^{\prime}$ lacproB, $I^{Q} A 16_{a m}-Z$ fusion; $\mathrm{Kn}^{\mathrm{R}}$; (Normanly et al. 1986), Gln $\Delta 114$ tRNA $^{\mathrm{Gln}}$ knockout strain is a derivative of the MG1655 E. coli strain and lacks the four tRNA $^{\text {Gln }}$ isoacceptors ( $\Delta g \ln X V W U$; W.H. McClain, unpubl.). The pGFIB plasmid is a high copy, ampicillin-resistant vector with efficient constitutive promoter and terminator ( $l p p$ and $r r n B$, respectively) for tRNA gene expression (Masson and Miller 1986); the pSAD plasmid is a medium copy, chloramphenicol-resistant vector with an $A r a C p B A D$ promoter that is induced by arabinose and repressed by glucose (Gabriel and McClain 2001); pGAD2 is a high-copy, ampicillin-resistant vector that contains the same AraCpBAD promoter as pSAD (Gabriel and McClain 2001); pSU81 is a medium-copy, chloramphenicol-resistant vector that contains a constitutive lpp promoter (Gabriel and McClain 2001).

\section{Construction of tRNA genes}

The construction of tRNA genes was performed in two ways, both employing the use of synthetic oligonucleotides. The six-piece construction method separates the tRNA into three regions: the 5' end, core region, and $3^{\prime}$ end. The regions of the tRNA were separated as follows: $5^{\prime}$ end: 1-13 (upper strand), 1-16 (lower strand); core region: 14-63 (upper strand), 17-58 (lower strand); 3 ' end: 64-76 (upper strand), 59-76 (lower strand). The restriction sites EcoRI and PstI were added to the $5^{\prime}$ and $3^{\prime}$ ends of the tRNA construct, respectively, as sticky ends. The oligonucleotides were obtained from either IDT or the UW-Biotech Center, and were resuspended in TE to a concentration of 20 pmole/ $\mu \mathrm{L}$. All oligonucleotides with the exception of the $5^{\prime}$ ends were phosphorylated, using PNK (New England Biolabs). Oligonucleotide (100 pmole) was combined with the phosphorylation reaction mixture and was incubated for $1 \mathrm{~h}$ at $37^{\circ} \mathrm{C}$. The PNK was inactivated at $65^{\circ} \mathrm{C}$ for 20 min. The DNA was then extracted with Bonner, centrifuged at maximum speed in a microfuge for $5 \mathrm{~min}$, and precipitated. The precipitated, phosphorylated DNA oligonucleotides were combined with the $5^{\prime}$ oligonucleotides and annealed by heating the mixture to $80^{\circ} \mathrm{C}$ for $5 \mathrm{~min}$ and slow cooling the mixture for 30 min. Once annealed, the tRNA gene construct was ligated into EcoRI and Pst I digested vector. The primary structures of tRNA genes were confirmed by DNA sequencing.

The one-step synthesis method has been described (Choi et al. 2002). The tRNA gene library for the GlnAGGU library experiment was constructed using this method. The synthetic oligonucleotides $5^{\prime}$-AAGCTTGAATCCNNATTGGGGTATCGCCAAG CGGTAAGGCACCGGTTTTTGATA-3' and 5'-GGATCCTGCA GNNNGATGGCTGGGGTACCTGGATTCGAACCAGGNNNNC CGGTATCAAAAACCGGT were obtained from IDT, and were used to construct a GlnAGGU tRNA gene, where N indicates a $25 \%$ mix in the four base variable loop. The oligonucleotides were then used in a one-cycle synthesis reaction, digested with EcoRI and PstI, and were ligated into the pGFIB plasmid. The tRNA gene library was tested in $G \ln \Delta 114$ knockout cells in a plasmid switch as described below. The total number of cells examined was $5 \times 10^{10}$ based on an appropriate dilution of a control sample, which gave 500 colonies per plate.

\section{Plasmid switches}

To prepare cells for a plasmid switch, an overnight culture of Gln $\Delta 114$ knockout cells supported by a wild-type tRNA ${ }^{\text {Gln }}$ gene carried on either the pSAD or pGAD2 maintenance plasmid was grown in MinA arabinose media (Gabriel and McClain 2001). The cells were then pelleted, washed three times in equal volume sterile $\mathrm{dH}_{2} \mathrm{O}$, and resuspended in $1 / 20$ volume sterile distilled $\mathrm{H}_{2} \mathrm{O}$. The cells were then separated into $20-\mu \mathrm{L}$ aliquots, and electroporated without added plasmid; this treatment reduces the intracellular pool of maintenance plasmid. The cells were allowed to recover for $20 \mathrm{~min}$ in SOC broth at $37^{\circ} \mathrm{C}$. The cells were then pelleted and washed as above. The appropriate test plasmid was added to the Gln $\Delta 114$ cells, which were then electroporated a second time. The cells were allowed to recover in SOC broth for $1 \mathrm{~h}$ at $37^{\circ} \mathrm{C}$. The cells were then subcultured into LB plus the appropriate antibiotic. Cells that lost the maintenance plasmid were found by picking and stabbing onto plates with the appropriate antibiotic. Loss of resistance to chloramphenicol (for pSAD) or ampicillin (for pGAD2) is evidence that the maintenance plasmid has been physically eliminated from the cell.

\section{Glucose strip test}

The glucose strip test has been described (Gabriel and McClain 2001; Moulinier et al. 2001). The plasmid pSU81 containing the appropriate test genes was transformed into $G \ln \Delta 114$ knockout cells. The cells were plated on MinA plates plus supplements, ampicillin and chloramphenicol, and were incubated at $37^{\circ} \mathrm{C}$ overnight. An individual colony was picked into $75 \mu \mathrm{L}$ of MinA without supplements, and was left to stand for $10 \mathrm{~min}$. A $0.8-\mu \mathrm{L}$ aliqot was then spotted onto a MinA plate plus supplements, arabinose, ampicillin, and chloramphenicol. The spot was streaked left to right across the plate in one continuous motion using a glass 
spreader. Once the streak was dry, a sterile paper strip saturated with $20 \%(\mathrm{w} / \mathrm{v})$ glucose was laid in the center of the plate, perpendicular to the streaks. The plate was incubated at $37^{\circ} \mathrm{C}$ overnight. Cells at the edge of the plate were supported by wild-type tRNA $^{\text {Gln }}$ expressed from the maintenance plasmid and possibly tRNA expressed from the test plasmid. Cells near the glucose strip will only be supported by the constitutively expressed test plasmid because maintenance plasmid gene expression is repressed by glucose.

\section{Northern blot analysis}

The Northern blot analysis method has been described (Gabriel and McClain 2001). The tRNA for Northern blot analysis was isolated by growing cells expressing the appropriate tRNA gene from a plasmid to an $\mathrm{OD}_{650}$ of 0.4 . Phenol was added directly to the growing culture, and left to shake for $10 \mathrm{~min}$. The culture was then centrifuged and the aqueous phase was precipitated with ethanol. The samples were dried and resuspended in TE. A $2-\mu \mathrm{L}$ aliquot was taken from the sample and heated at $65^{\circ} \mathrm{C}$ for $5 \mathrm{~min}$. The sample was then loaded and electrophoresed on a $10 \%$ polyacrylamide gel containing $7 \mathrm{M}$ urea. The samples were electroblotted onto Nytran SuperCharge membrane for $45 \mathrm{~min}$. The membrane was hybridized to $\left(5^{\prime}-{ }^{32} \mathrm{P}\right)$-labeled probe at a hybridization temperature of $50^{\circ} \mathrm{C}$. The probes for most tRNAs were complementary to tRNA ${ }^{\text {Gln }}$ positions 29-47; that for GlnQSerC44 covered residues $29-48$.

\section{ACKNOWLEDGMENTS}

This work was supported by NIH Grant GM42123.

The publication costs of this article were defrayed in part by payment of page charges. This article must therefore be hereby marked "advertisement" in accordance with 18 USC section 1734 solely to indicate this fact.

Received August 22, 2003; accepted September 22, 2003.

\section{REFERENCES}

Altman, S. 1971. Isolation of tyrosine tRNA precursor molecules. Nat. New Biol. 229: 19-21.
Bullock, T.J., Sherlin, L.D., and Perona, J.J. 2000. Tertiary core rearrangements in a tight binding transfer RNA aptamer. Nat. Struct. Biol. 7: 497-504.

Cerchia, L., Hamm, J., Libri, D., Tavitian, B., and de Franciscis, V. 2002. Nucleic acid aptamers in cancer medicine. FEBS 528: 12-16.

Choi, H.C., Otten, S., and McClain, W.H. 2002. Isolation of novel tRNA $^{\text {Ala }}$ mutants by library selection in a tRNA ${ }^{\text {Ala }}$ knockout strain. Biochimie 84: 705-711.

Comer, M.M., Guthrie, C., and McClain, W.H. 1974. An ochre suppressor of bacteriophage T4 that is associated with a transfer RNA. J. Mol. Biol. 90: 665-676.

Famulok, M., Mayer, G., and Blind, M. 2000. Nucleic acid aptamersFrom selection in vitro to applications in vivo. Acc. Chem. Res. 33: 591-599.

Gabriel, K. and McClain, W.H. 2001. Plasmid systems to study RNA function in Escherichia coli. J. Mol. Biol. 310: 543-548.

Gold, L., Polinsky, B., Uhlenbeck, O., and Yarus, M. 1995. Diversity of oligonucleotide functions. Annu. Rev. Biochem. 64: 763-797.

Khvorova, A., Kwak, Y., Tamkun, M., Majerfeld, I., and Yarus, M. 1999. RNAs that bind and change the permeability of phospholipid membranes. Proc. Natl. Acad. Sci. 96: 10649-10654.

Masson, J. and Miller, J.H. 1986. Expression of synthetic suppressor tRNA genes under the control of a synthetic promoter. Gene 47: 179-183.

McClain, W.H. and Seidman, J.G. 1975. Genetic perturbations that reveal tertiary conformation of tRNA precursor molecules. Nature 257: 106-110.

- 1987. Genetic conversion of G-C base-pairs to A-U basepairs in transfer RNA. J. Mol. Biol. 197: 605-608.

McClain, W.H., Jou, Y., Bhattacharya, S., Gabriel, K., and Schneider, J. 1999. The reliability of in vivo analysis of tRNA aminoacylation. J. Mol. Biol. 290: 391-409.

Moulinier, L., Eiler, S., Eriani, G., Gangloff, J., Thierry, J.C., Gabriel, K., McClain, W.H., and Moras, D. 2001. The structure of an AspRS-tRNA ${ }^{\text {Asp }}$ complex reveals a tRNA-dependent control mechanism. EMBO J. 20: 5290-5301.

Nissan, T.A., Oliphant, B., and Perona, J.J. 1999. An engineered class I tRNA with a class II tertiary fold. RNA 5: 434-445.

Normanly, J., Masson, J., Kleina, L.G., Abelson, J., and Miller, J.H. 1986. Construction of two Escherichia coli amber suppressor genes: tRNA ${ }^{\text {Phe }}$ CUA and tRNA ${ }^{\text {Cys }}$ CUA. Proc. Natl. Acad. Sci. 83: 65486552.

Seidman, J.G., Comer, M.M., and McClain, W.H. 1974. Nucleotide alterations in the bacteriophage T4 glutamine transfer RNA that affect ochre suppressor activity. J. Mol. Biol. 90: 677-689.

Stubenrauch, M. 1996. "In vitro selection and characterization of tRNA-like substrates of E. coli glutaminyl-tRNA synthetase." Ph.D. thesis, University of Colorado, Boulder, CO. 

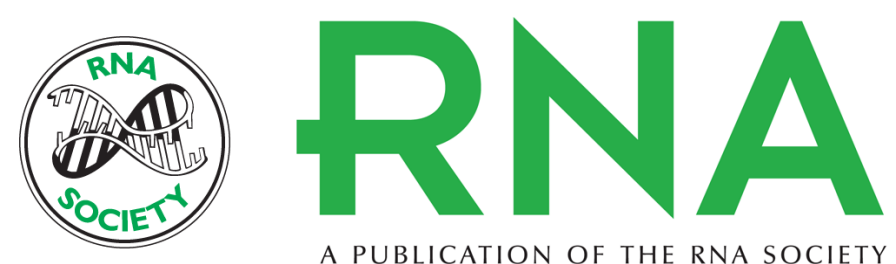

A PUBLICATION OF THE RNA SOCIETY

\title{
Aptamer redesigned tRNA is nonfunctional and degraded in cells
}

\author{
DENNIS LEE and WILLIAM H. MCCLAIN
}

RNA 2004 10: 7-11

References This article cites 17 articles, 3 of which can be accessed free at:

http://rnajournal.cshlp.org/content/10/1/7.full.html\#ref-list-1

\section{License}

Email Alerting Receive free email alerts when new articles cite this article - sign up in the box at the Service top right corner of the article or click here.

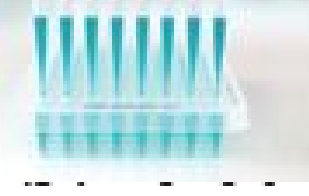

